\title{
Thermographic findings of spinal cord tumors
}

by Ho Yeol Zhang

\author{
Neurosurgery, National Health Insurance Service Ilsan Hospital, Yonsei University College of \\ Medicine, Korea, hoyeolzhang@gmail.com
}

\begin{abstract}
We analyzed IR thermographies of 137 spinal cord tumor patients. Thermography $(+)$ with level correlation was $67.8 \%$, symptom correlation was found $28.8 \%$ with local pain, $81.1 \%$ with radiculopathy. Myelopathy $(+)$ thermography was $71.9 \%$ in cervical level, $61.5 \%$ in thoracic, $75.6 \%$ with para(quadri)paresis and over $100 \%$ with Brown-Sequard syndrome. For the extradural or IDEM tumors, thermography represented the thermatomal demarcation in the dermatome and below well for myelopathy patients. In the intramedullary tumors, thermography frequently showed negative findings. Thermatomal hypothermia is a useful landmark for the differential diagnosis of schwannoma and meningioma or cauda equina and conus medullaris tumor also.
\end{abstract}

\section{Introduction}

Since Lawson (1) reported the clinical application of thermography in 1956, the infrared (IR) thermographic imaging technique has been used for the diagnosis, treatment effect, or pain intensiveness of various medical diseases. There are many papers on IR thermographic findings in disc herniation since Pochaczvsky (2) reported the usefulness in radiculopathy (3 5). The use of a computerized and digitalized IR thermography instead of the contact type liquid thermography in Korea was started at the Department of Neurosurgery of the Gangnam Severance Hospital in the year 1990 (6).

The reason why it is used in spinal diseases is that in the case of radiculopathy the temperature of the nerve root distributional area changes. This can be used in the diagnosis of various spinal diseases, the severity of the pain and its level of recovery. In the spinal cord tumor cases, IR thermography may be also useful to detect the painful areas or neurologic symptoms, because most of these are arisen from spinal nerve and disturb the neurologic homogeneity of symptomatic area of human body.

This paper is focused on the correlation among the IR thermographic findings and the patients' neurologic, radiologic symptoms and pathologic findings. The objective of this study is to find out the usefulness of IR thermography for the diagnosis and pathologic classification of spinal cord tumors.

\section{Materials and Methods}

We reviewed the records of patients, who underwent surgical treatment because of spinal cord tumors between 1995 and 1999, 137 patients were included. Spinal cord tumor can be classified as the extradural (ED) tumor, intradural extramedullary (IDEM) tumor, and intramedullary (IM) tumor by the topical relationship with dura and spinal cord itself (Fig. 1).

Physical and neurologic examinations can be divided as the local pain, radiculopathy, and myelopathy. The local pain means the pain around the local vertebrae (cervical, thoracic or lumbar) or paravertebral muscle pain. In the case where local pains exist, (+) IR thermographic findings were defined as a case in which a hyperthermia is located at the local paravertebral painful area. In the case where radiculopathy exists, $(+)$ IR thermographic findings were defined as a case in which the hypothermia is shown along the involved nerve distributional thermatome. While in the case where myelopathy exists, if the patients' symptom was paraparesis or qaudriparesis, (+) IR thermographic findings were defined as hypothermia below the lesion, while it was defined as ipsilateral hypothermia when the patient was showing the Brown-Sequard Syndrome.

\section{Results}

\subsection{ED Tumors}

There were 31 patients with ED spinal cord tumor. When the anatomical location of the tumor was compared to the findings of IR thermography, 10 out of the 11 patients with cervical tumor was thermography (+), 8 out of the 13 thoracic tumor patients was thermography $(+)$, and 6 of the 7 lumbar tumor patients was thermography $(+)$. Overall, 24 out of 31 extradural spine tumor patients $(77.4 \%)$ were thermography $(+)$.As for the relationship between the patient's symptom and the IR thermography, thermography $(+)$ was shown in 8 of 18 local pain patient, 11 of 17 radiculopathy patients, 6 of 8 myelopathy (para/quadriparesis) patients and in all 4 patients with the Brown-Sequard syndrome (figure 1).

\subsection{IDEM tumors}

Most of the IDEM tumor was schwannoma and meningioma, and in rare cases arachnoid cyst. Because schwannomas originate from the nerve roots while meningiomas originate from the arachnoid cell nest, the thermographic recordings were analyzed separately.

\subsubsection{Schwannoma}

33 patients with schwannoma were enrolled in this study. The results according to their anatomical site of tumor and IR thermography showed a $78.6 \%$ chance of thermography $(+)$ for the cervical tumor and a $73.7 \%$ 
thermography $(+)$ for the thoracic tumor. For the relationship between the symptom and IR thermography, local pain showed thermography $(+)$ with a $53.8 \%$ chance and radiculopathy had a $78.6 \%$ chance while the chance was $60.0 \%$ for patients with myelopathy (para/quadriparesis) and $77.8 \%$ for the Brown-Sequard syndrome (figure 2).

\subsubsection{Non-Schwannoma (meningioma, archnoid cyst)}

In 16 patients with non-schwannoma, 15 patients were diagnosed with meningioma and 1 patient was diagnosed with arachnoid cyst. For the relevance between their anatomical site of tumor and finding of IR thermography, 1 out of 3 patients with cervical tumor was thermography (+) and 7 out of $13(53.8 \%)$ patients with thoracic tumor was thermography $(+)$. As for the relationship between their symptom and IR thermography, thermography $(+)$ was not showed in patients with local pain and radiculopathy. However, thermography $(+)$ was shown in $60.0 \%$ of the cases with myelopathy (para/quadriparesis) and Brown-Sequard syndrome. Three out of 15 patients showed para(quadri)paresis and 6 out of 15 showed Brown-Sequard syndrome (figure 3).

\subsection{IM tumors}

11 patients with IM tumor (such as astrocytoma and ependymoma) were enrolled in this study. The results according to their anatomical site of tumor and IR thermography showed that 1 out of 4 patients with cervical tumor was thermography (+) and that 3 out of 7 thoracic tumor patients were thermography (+). Overall, 4 out of 11 patients $(36.3 \%)$ with IM spinal cord tumor were thermography $(+)$. For the relationship between the symptom and IR thermography, thermography $(+)$ was not shown in patients with local pain or radiculopathy. However, thermography $(+)$ was shown in 4 out of 9 patients $(44.4 \%)$ with myelopathy (para/quadriparesis) (figure 4$)$.

\subsection{Cauda equina tumor}

Cauda equina tumor occurs in places where only nerve roots exist, therefore the tumor is almost a schwannoma and in rare cases a meningioma. For the relevance between the symptom and IR thermography, thermography $(+)$ was $30.8 \%$ in cases with local pain, $95.2 \%$ in cases with radiculopathy on unilateral leg, $47.4 \%$ in cases with a radiculopathy on both legs. Overall, 29 out of 40 patients $(72.5 \%)$ with cauda equina tumor were thermography $(+)$ (figure 5).

\subsection{Conus medullaris tumor}

Only 6 patients were diagnosed with the conus medullaris tumor. Most of them complained paralysis in their lower extremities. For the relation between their symptoms and IR thermography, only $40.0 \%$ of the patients with a radiculopathy on both legs showed thermography $(+)$. Overall, 2 of 6 patients $(33.3 \%)$ with conus medullaris tumor were thermography $(+)$ (figure 6).

\subsection{Overall results}

All of 137 patients' results were summarized. IR thermography (+) finding with level correlation was found $67.8 \%$ among the all spinal cord tumors. Among these, in the cervical spinal level were $71.9 \%, 61.5 \%$ in thoracic, and $71.7 \%$ in lumbar levels (Table 11 ).

IR thermography (+) finding with symptom correlation was found $28.8 \%$ with local pain, $81.1 \%$ with radiculopathy, $75.6 \%$ with para(quadri)paresis and over $100 \%$ with Brown-Sequard syndrome

\section{Discussion}

Among the various spinal diseases, herniated lumbar disc (6 9), herniated cervical disease (10) or whiplash injury (11) are known to the well visualization of the painful site with good symptomatic correlations. But there are rare reports about IR thermography of the spinal cord tumor. Only 4 papers can be found from the search of PubMed. The first report was done by Goldberg et al at 1966 (12), and other three are from Russia (13 15). These four are not available of full text or English version through PubMed.

Our paper is analyzed 137 spinal cord tumor patients. The thermographic characteristics are described with the thermographic expression rate of the related neurologic symptoms.

In our series, ED tumors are mostly spinal metastatic cases and 3 cases of osteoma. IR thermography $(+)$ rate was $77.4 \%$. Among this result, IR thermography $(+)$ of local pain is only $44.4 \%$. It is thought to be returned symmetric thermal distribution very quickly from the thermal asymmetry because of the many musculatures supplied from various nerve roots. Zhang et al (16) reported the thermal asymmetry of low back area is higher incidence in the shorter duration of illness (herniated lumbar disc disease), and lower incidence in the chronic duration of illness (spondylolysis, spondylolisthesis). In the acute state, thermal asymmetry on the lower back is presented by the dorsal ramus from the involved nerve root, but soon after illness, many back muscles from various uninvolved nerve root supply (trapezius, rhomdoid, lattissimus dorsi, erector spinae, etc) compensate the thermal asymmetry of the lower back. Like these mechanisms, thermographic visualization of the local pain was low incidence than neurological symptoms, because most of spinal cord tumor has the longer duration than herniated lumbar disc. As this reason, ED, IDEM, IM, cauda equina and conus medullaris tumors are low incidence of local pain also.

Radiculopathy (+) thermographic findings were $64.7 \%$, even though ED tumor is not originated from the nervous cell origin. In 1940, Batson (17) theorized arterial deposits of metastatic cells would occur near the endplates, whereas the venous vascular anatomy of the spine would result in metastases in the central or posterior vertebral body. Nowadays, the mechanism of tumor spread to the vertebral body may not be via a pure arterial or 
venous route. Other mechanisms such as tissue specificity, cascade system, and closed loop circulation system may be involved (18). In our series, based on the Batson's theory, the hematogenous tumor metastasis is settled down the vertebral endplate. So, various tumors are inoculated and grew around the disc space and their growth became to compress the nerve root firstly, then radiculopathy symptoms and IR (+) radiculopathy pattern could occur frequently $(64.7 \%)$. And tumor grew enough to compress the spinal cord parenchyma, it could show the thermographic myelopathy findings like as thermatomal demarcation, para(quadri)paresis and Brown-Sequard syndrome.

In the IDEM tumors, we divided it Schwannoma and non-Schwannoma cases. Because schwannoma originates from the nerve sheath cell, myelin which covers nerve root, the physiologic change of the nerve root was reflected in the thermatomal hypothermia well. Radiculopathy $(+)$ thermographic findings were the highest $(78.6 \%)$ in schwannoma cases. On the other hand, because the non-Schwannoma tumors do not originate from the neural cell, there was almost no radiculopathy and because it is not an actual physiologic change, changes in thermatomal hypothermia did not occur also. However, in cases where a large tumor provoked a myelopathy symptom, the cord compression due to this mass showed the same patterns of thermatomal hypothermia regardless of the type of tumor. If we meet the IDEM tumor's MRI, it is difficult to differentially diagnose the schwannoma or meningioma. If we take a thermography at this time, we can imagine the diagnosis more precisely whether it is schwannoma (radiculopathy expression rate was $78.6 \%$ ), or meningioma $(0 \%$ of radiculopathy).

In the IM tumors, there was one of the least thermography $(+)$ rate $(36.3 \%)$ among all kinds of tumors. Conus medullaris is also a kind of spinal cord itself. So it is also the least thermography $(+)$ rate $(33.3 \%)$. IM tumors were presented as the para(quadric)paresis in most cases because it compressed spinal cord parenchyma and make a myelopathy symptom only. So the thermography (+) findings could be found only in para(quadric)paresis or Brown-Sequard syndrome. Especially in the conus medullaris tumors, myelopathy is almost bladder, bowel problems. Our protocol of the patient's position to take the IR thermography is standing only. We don't take the IR thermography of patient's inguinal area for the privacy problems. If the conus medullaris tumor is big enough to involve the lumbar spinal cord (located about T11/12 vertebra level) extended from the end of spinal cord, then it could be found the leg thermal asymmetry.

Level L1 is when the end of the spinal cord gets smaller and turns in to a conus medullaris. Then the section, around the conus medullaris, from $\mathrm{T} 12$ to $\mathrm{L} 1 / 2$ is where the innumerable nerve roots originate. If tumor arises in this area, it is impossible to differentiate through the MRI whether the origin of the tumor is the conus medullaris or the cauda equina. If the main symptom in this case is radiculopathy, the cauda equina lesion is to be suspected as the origin of tumor. On the other hand, if the main symptom is myelopathy (bladder/bowel symptom), the conus medullaris lesion may be suspected. In the case of IR thermography, if it is thermography $(+)$, cauda equina tumor (especially schwannoma), can be suspected. For the conus medullaris tumors that occur in the intramedullary portion of the spinal cord, the symptom is localized to the bladder, bowel and inguinal area, therefore is thought to have a high possibility of being thermography (-), a non-specific pattern. Therefore, if the spinal cord is located in the T12 L1/2 area, MRI could not differentially diagnose it from conus medullaris or cauda equina origin tumor frequently. But if we use the IR thermography, it can be more easily differentiate due to the good visualization of radiculopathy pattern in the cauda equina tumor.

In overall results, Brown-Sequard syndrome is higher incidence (over 100\%) than patients' neurologic signs by the doctor's examinations. This means IR thermography could detect the thermal asymmetry precisely, even though patient didn't feel or doctors didn't detect the left-right asymmetric body condition. The symptoms of myelopathy, radiculopathy from the spinal cord tumors can be detected easily by the IR thermography, but local pain was not well visualized.

\section{Conclusion}

In the spinal cord tumor cases, IR thermography reveals various neurologic symptoms depending on the location, size, and pathology of tumor. For the ED tumors or IDEM tumors which have a large enough size to cause myelopathy, the IR thermography represented the hypothermia (thermatomal demarcation) taking place in the dermatome and below well for para(quadric)paresis patients and also represented the Brown-Sequard syndrome well, showing a high relevance to the patient's symptoms. As the schwannoma originates from the nerve roots, when it shows radiculopathy as its symptoms, thermatomal hypothermia is exceptionally well seen in IR thermography. So in cases of reasonably sized tumors which cause radiculopathy, the IR thermography can be used to determine whether it is a schwannoma or a meningioma among the IDEM tumors. In cases of IM tumor, the IR thermography frequently showed negative findings although the patient was suffering from myelopathy, a finding common in patients with a mild paralysis. Thermatomal hypothermia is a useful landmark for the differential diagnosis of the cauda equina tumor and the conus medullaris tumor. Although IR thermography currently is not used as a tool to diagnose the spinal cord tumor, it could be a useful tool in recognizing the severity of the symptom and in the differential diagnosis of tumors as it represents neurologic symptoms very well.

\section{REFERENCES}

[1] Lawson R., "Implications of surface temperature in the diagnosis of breast cancer". Canadian Medical Association Journal, vol. 75, pp. 309-310, 1956.

[2] Pochaczvsky R., Wexler CE., Meyers PH., Epstein JA., Marc JA., "Liquid crystal thermography of the spine 
and extremities. Its value in the diagnosis of spinal root syndromes". Journal of Neurosurgery, vol. 56, pp. 386-95,1982.

[3] Uematsu S., Edwin D.H., Jankel W.R., Kojikowski J., Trattner M., "Quantification of thermal asymmetry. Part 1: Normal values and reproducibility”. Journal of Neurosurgery, vol. 69(4), pp. 552-555,1988.

[4] Uematsu S., Jankel W.R., Edwin D.H., Kim W., Kojikowski J., Rosenbaum A., Long D.M., "Quantification of thermal asymmetry. Part 2: Application of low back pain and sciatica”. Journal of Neurosurgery, vol. 69(4), pp. 556-61,1988

[5] Mills G.H., Davie G.K., Getty G.J.M., Conway J., "The evaluation of liquid crystal thermography in the investigation of nerve root compression due to lumbosacral lateral spinal stenosis". Spine, vol. 11, pp. 427-32, 1986.

[6] Kim Y., Cho Y., Oh S., "Digital infrared thermographic imaging (D.I.T.I.) in herniated lumbar disc patients". Journal of Korean Neurosurgical Society, vol. 19(10), pp. 1303-13,1990.

[7] Kim Y., Cho Y., Zhang H., "Thermatome of the lumbosacral nerve roots". Journal of Korean Neurosurgical Society, vol. 24, pp. 33-46,1995

[8] Cho Y., Kim Y., Zhang H., "Clinical efficacy of digital infrared thermographic imaging in multiple lumbar disc herniations". Journal of Korean Neurosurgical Society, vol. 27, pp. 237-45.1998.

[9] Zhang H., Chin D., Cho Y., Kim Y., "Correlation between pain scale and intrared thermogram in lumbar disc herniations". Journal of Korean Neurosurgical Society. vol. 28, pp. 253-58,1999.

[10] Zhang H., Kim Y., Cho Y., "Thermatomal changes in cervical disc herniations". Yonsei Medical Journal, vol. 40, pp. 401-12,1999.

[11] Zhang H., Cho B., Kim H., Cho Y., "Thermographic diagnosis of whiplash injury with/without radiculopathy". Key Engineering Materials, vol. 321-23, pp. 845-48, 2006.

[12] Goldberg H.I., Heinz E.R., Taveras J.M., "Thermography in neurological patients. Preliminary experiences". Acta radiologica: diagnosis (Stockh), vol. 5, pp. 786-95, 1966

[13] Fraerman A.P., Kolesov S.N., Likhterman L.B., "Diagnostic possibilities and prospects for the use of thermovision in neurosurgical clinical practice". Voprsy Neirokhirrugii, vol. 2, pp. 27-35, 1978.

[14] Mel'nikova V.P., Nikiforov B.M., Voronov V.G., "Thermography in the diagnosis of spinal cord tumors". Zhurnal Nevropatologi Psikhiatrii Imeni S S Korsakova, vol. 79(5), pp. 555-59,1979.

[15] Likhterman L.B., Kolesov S.N., Kisliakov A.G., Fedoseev L.I., Lebskiĭ luV, "Diagnostic thermoradiolocation in neurosurgery". Zhurnal Voprosy Neirokhirurgii Imeni N N Burdenko, vol. 4, pp. 19-25,1986.

[16] Zhang H., Cho Y., Kim Y., "Thermal asymmetry of the low back area in degenerative lumbar diseases". Korean Journal of Thermology, vol. 7, pp. 69 73, 2006.

[17] Batson O.V., "The function of the vertebral veins and their role in the spread of metastases". Annals of Surgery, vol. 12(1), pp. 138-49, 1940.

[18] Yuh W.T., Quets J.P., Lee H.J., Sinonson T.M., Michalson L.S., Nguyen P.T., Sato Y., Mayr N.A., Berbaum K.S., "Anatomic distribution of metastases in the vertebral body and modes of hematogenous spread". Spine, vol. 21(19), pp. 2243-50,1996. 

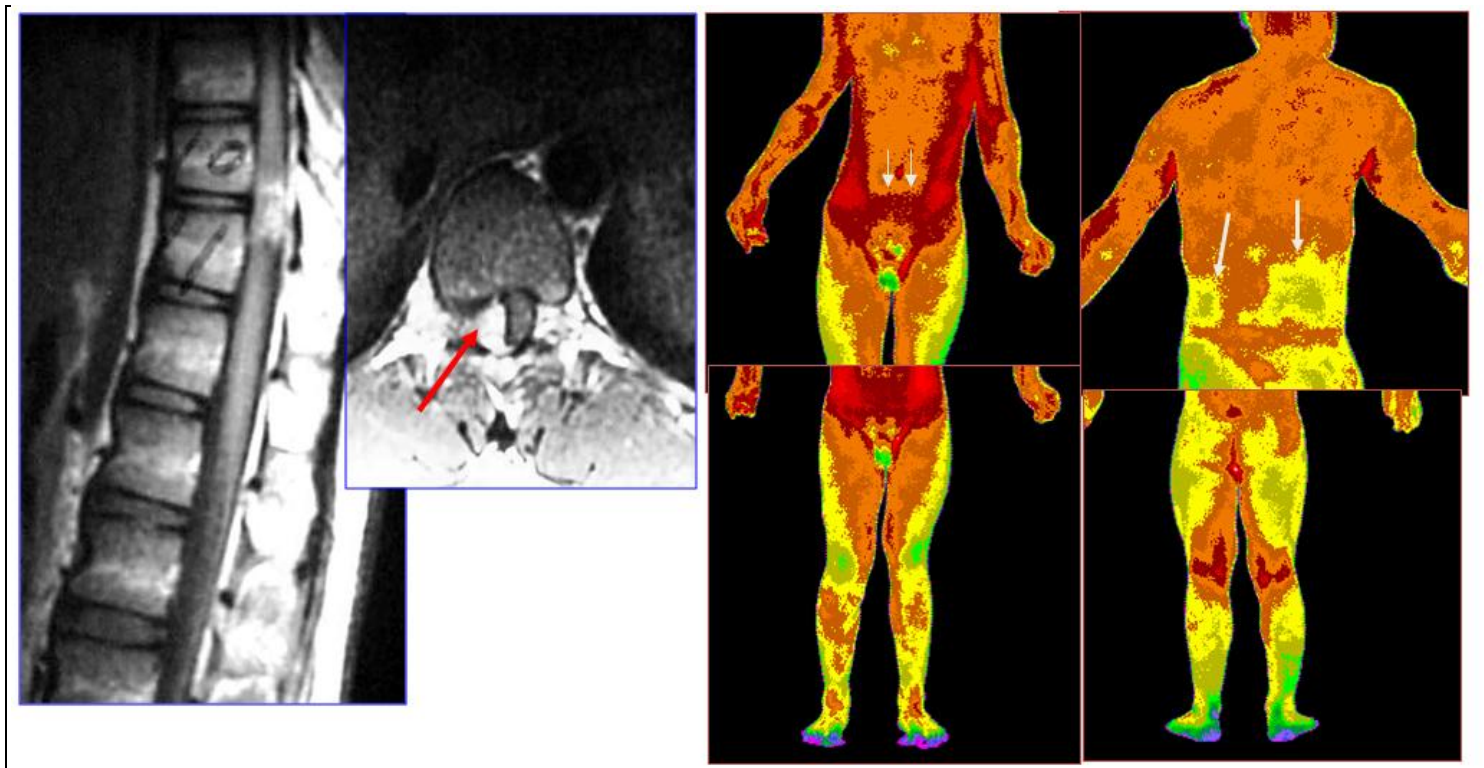

Fig. 1. ED tumor

The diagnosis was osteoma at T10/11 area, right side extradural spinal cord tumor. Patient's symptom was paraparesis grade 4 and sensory loss below T11 dermatome. IR thermography showed sudden thermal change (thermatomal demarcation) at T11 dermatome.

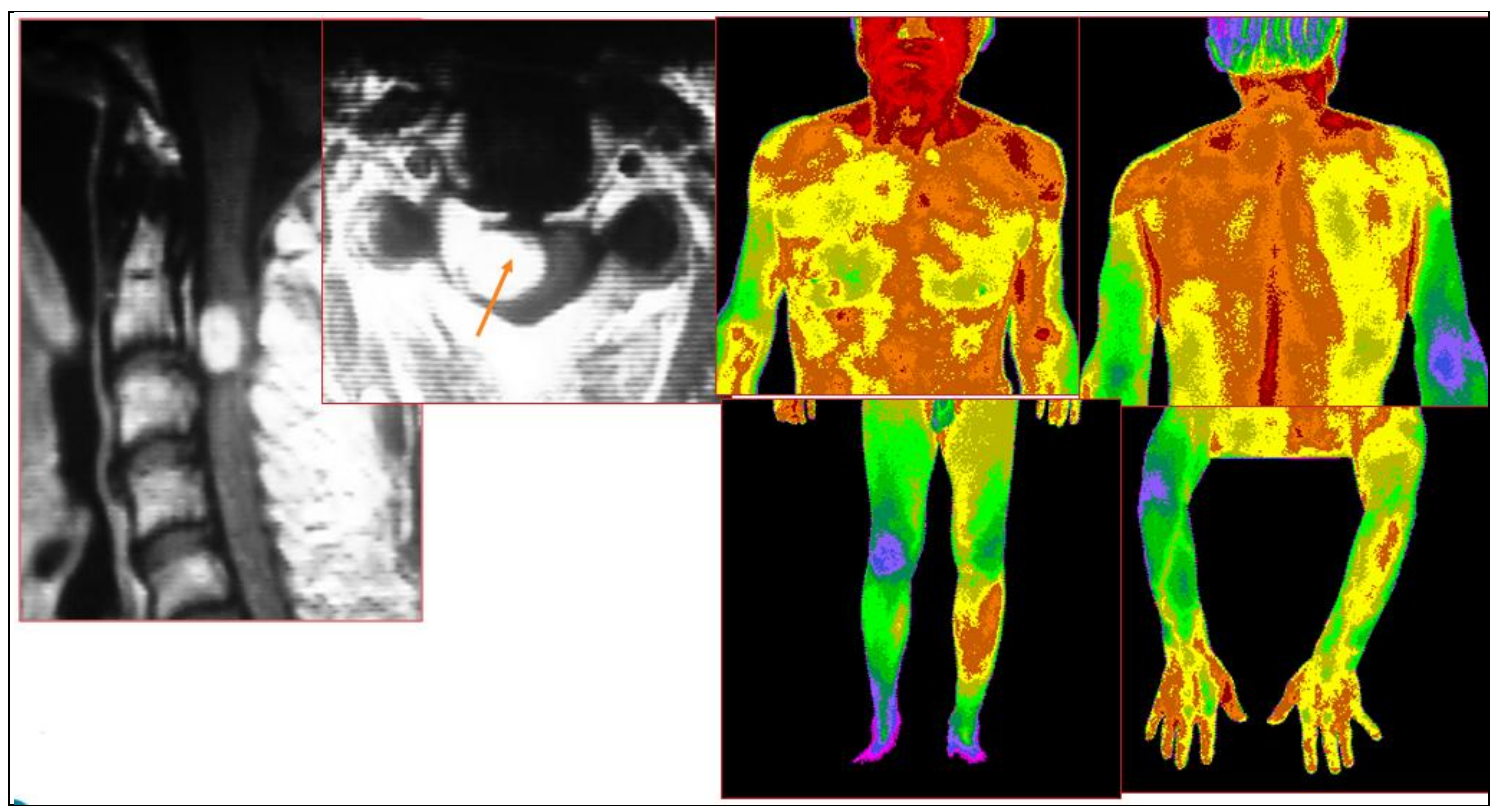

Fig. 2. IDEM tumor with myelopathy, especially Brown-Sequard syndrome.

Patient's symptom was neck pain, right side weakness and left side sensory deficit. Diagnosis was Schwannoma, C1/2, right side. IR thermography showed hyperthermia on the right side posterior neck muscle, right side (motor weakness side, tumor side) hypothermia on the body, arm, leg. 


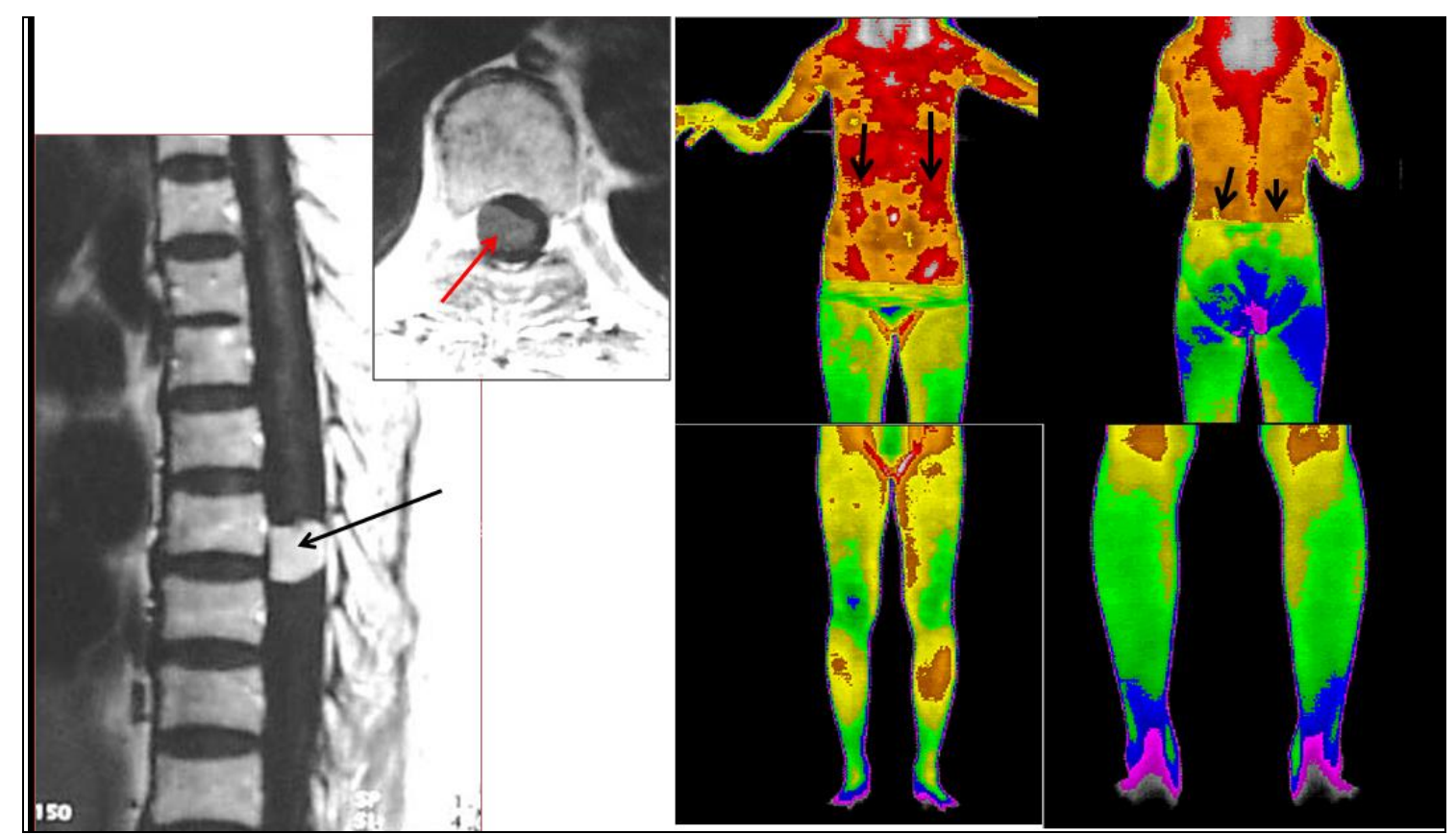

Fig. 3. IDEM tumor, meningioma case.

MRI showed T7/8 right side tumor. Her symptom was right side leg subjective weakness and both leg decreased sensation. IR thermography showed sudden hypothermia (thermatomal demarcation) about T8 dermatome and also right leg, buttock is more hypothermia than left side (Brown-Sequard syndrome).

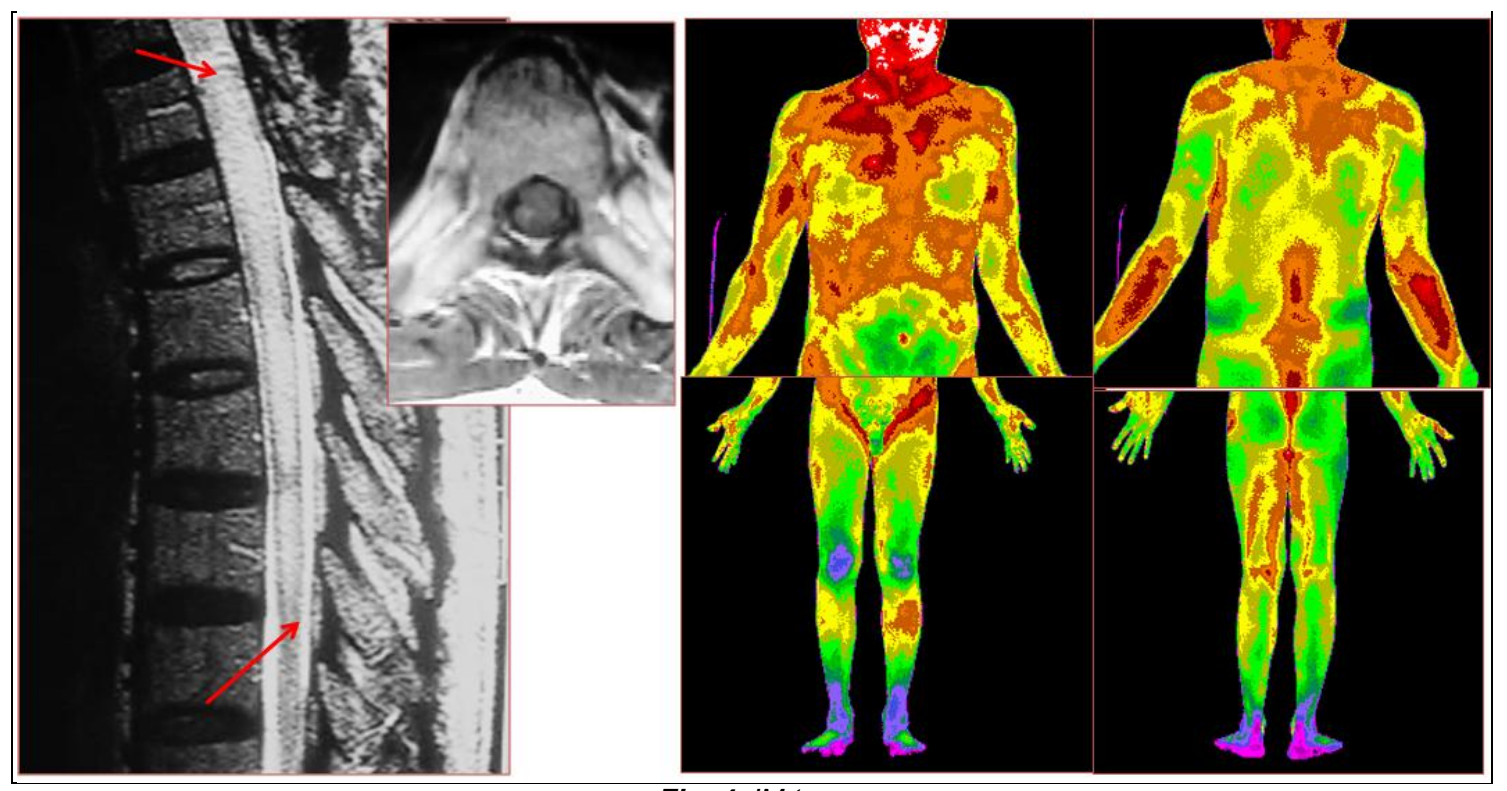

Fig. 4. IM tumor.

MRI showed intamedullary tumor from T2 to T6/7 level. Pathologic diagnosis was ependymoma. This patient complained dorsal pain, both leg numbness, and intermittent both leg weakness. IR thermography (+) finding was right leg hypothermia. 


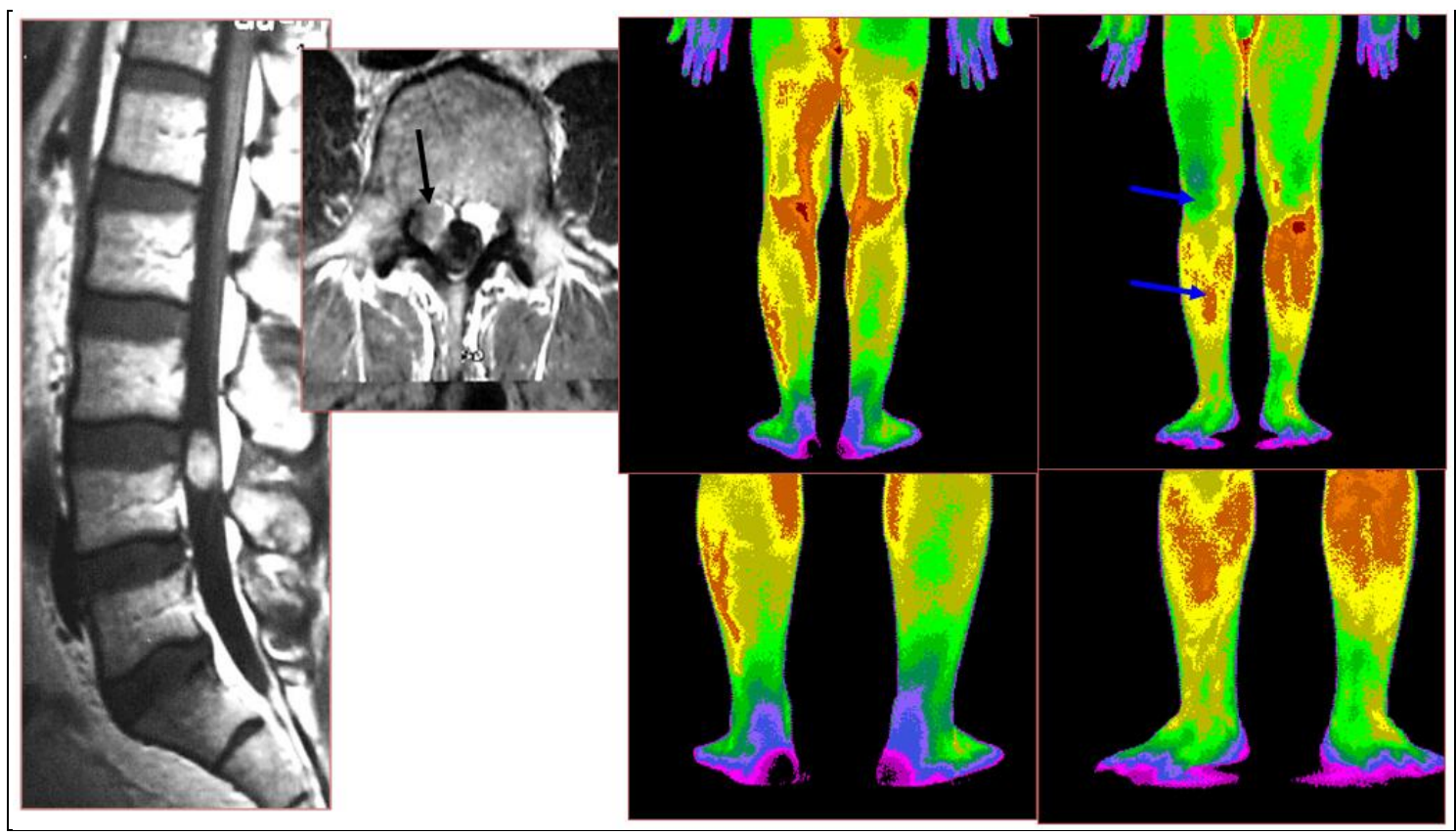

Fig. 5. Cauda equine tumor

MRI showed L4 right side tumor. Pathologic diagnosis was Schwannoma at right L4 nerve root. Patient's symptom was right leg pain. IR thermography $(+)$ finding was right $L 4$ thermatome hypothermia.

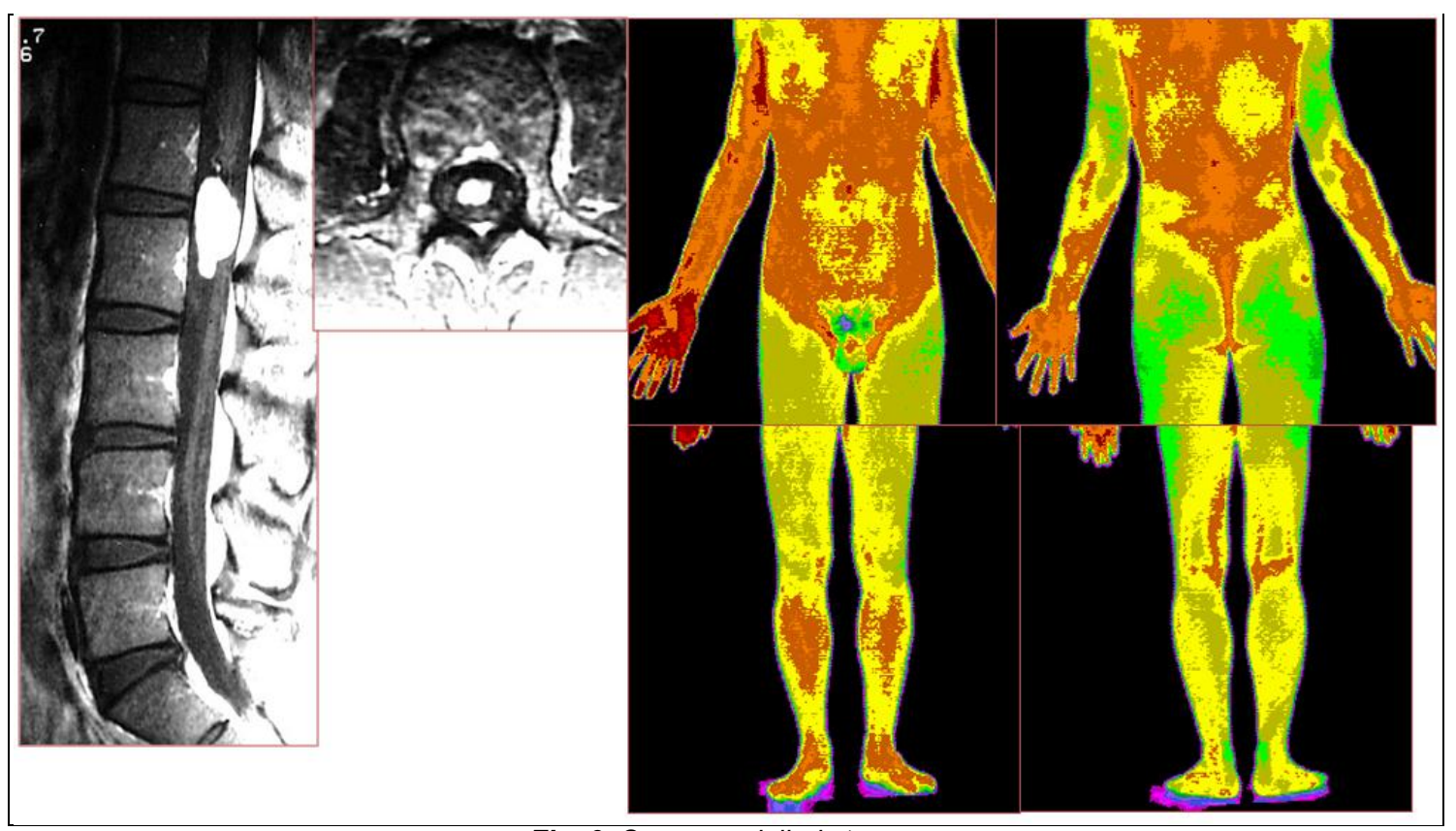

Fig. 6. Conus medullaris tumor

MRI showed the highly gadolinium enhanced mass at the end of the spinal cord L1/2 area. Patient's symptom was low back pain, both leg pain (left is more painful) for 1.5 years. IR thermography findings were non-specific or negative finding. 